\title{
NUMERICAL MODELLING OF METAL-ELASTOMER SPRING NONLINEAR RESPONSE FOR LOW-RATE DEFORMATIONS
}

\author{
Wojciech SIKORA*, Krzysztof MICHALCZYK", Tomasz MACHNIEWICZ ${ }^{* *}$ \\ *Faculty of Mechanical Engineering and Robotics, Department of Machine Design and Technology, \\ AGH University of Science and Technology, al. Mickiewicza 30, 30-059 Kraków, Poland \\ ${ }^{* *}$ Faculty of Mechanical Engineering and Robotics, Department of Strength and Fatigue of Materials and Structures, \\ AGH University of Science and Technology, al. Mickiewicza 30, 30-059 Kraków, Poland \\ wosikora@agh.edu.pl, kmichal@agh.edu.pl, machniew@agh.edu.pl
}

received 6 June 2017, revised 28 February 2018, accepted 5 March 2018

\begin{abstract}
Advanced knowledge of mechanical characteristics of metal-elastomer springs is useful in their design process and selection. It can also be used in simulating dynamics of machine where such elements are utilized. Therefore this paper presents a procedure for preparing and executing FEM modelling of a single metal-elastomer spring, also called Neidhart's spring, for low-rate deformations. Elastomer elements were made of SBR rubber of two hardness values: $50^{\circ} \mathrm{Sh}$ and $70^{\circ} \mathrm{Sh}$. For the description of material behaviour the Bergström-Boyce model has been used.
\end{abstract}

Key words: Bergström-Boyce Model, Neidhart's Spring, Metal-Elastomer Spring, FEM, SBR Rubber, Nonlinear Spring

\section{INTRODUCTION}

Nowadays, there are many different solutions for elastic elements utilized in machine design. Most basic and well known are metal springs, however there are also other alternatives. One of them is metal-elastomer spring based on Neidhart patent (Neidhart, 1951). This type of spring can be - compared to metal springs - advantageous in some applications due to significant damping properties. It relates in particular to vibrating machines, in which resonant vibrations amplitudes during run-up and rundown phase pose important problem (Cieplok, 2009). Application range of this type of metal-elastomer springs in various machines and industries constantly grows over the years. This makes justified to conduct research on them, which will lead to better understanding how they function and allow to improve their design.

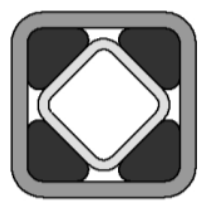

a)

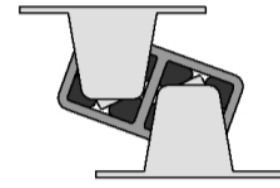

b)

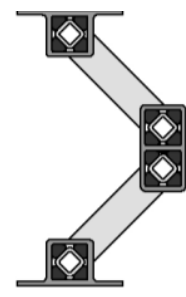

c)
Fig. 1. Selected constructions of Neidhart's spring assemblies: a) single joint, b) two-joint support, c) four-joint support

Fig. 1 shows three different constructions of spring based on Neidhart patent. Single metal-elastomer spring shown in Fig. 1a is only capable of angular deformation, whereas two other constructions (Fig. 1b and 1c) are able to undergo linear deformation of large values, what makes them a significant competition even for helical springs. Moreover, by modifying rubber elements geometry or by changing rubber blend it is possible to change the mechanical properties of such system. Flexibility in their design proves the great potential of the metal-elastomer springs.

Proper selection of geometrical and material properties of metal-elastomer spring is essential in terms of Its application and faultless operation. To the best knowledge of authors, currently there is no analytical model in available literature, which would allow to describe relation of spring geometry and material properties of rubber with mechanical characteristics of metalelastomer spring based on Neidhart patent. Probably the main reason behind this is that rubber parts undergo significant deformations during initial assembly and later operation of the spring. Moreover, nonlinear character of stress-strain relation of the elastomer itself also pose difficulties. Faced with described circumstances finite element method (FEM) seems to be a reasonable solution for modelling of this type of springs.

This paper presents an approach to modelling of a metalelastomer spring using FEM. The first part describes and analyses Bergström-Boyce material model which was used to describe mechanical properties of the elastomer blends and was later utilized within FEM software. In the second part of this work the experimental tests conducted to receive empirical characteristics of metal-elastomer springs for given types of rubber have been presented. The results obtained were then used to assess accuracy of FEM modelling.

\section{MATERIAL MODEL}

Most of the well-known classic material models like MooneyRivlin (Mooney, 1940), Yeoh (1993), Gent (1996) or Arruda-Boyce (Arruda and Boyce, 1993) and others are sufficient when it comes 
to modelling static or quasi-static loading of an elastomer in typical laboratory tests with load modes like uniaxial tension, compression or simple shear. They are still commonly used and appear in literature (Diego et. al., 2017; Sikora, 2016; Ghoreishy et al., 2015). However, in practical applications, elastomer components usually undergo full cycles of loading and unloading, e.g. cylindrical rubber springs used as support in vibrating screens or feeders, displaying elastic hysteresis. Problem which arises is how to describe such behaviour, resulting from elastomer viscosity and connected with its time-dependent deformation. Mentioned above difficulty is still not completely solved and most of presented solutions are more empirical-based than analytical.

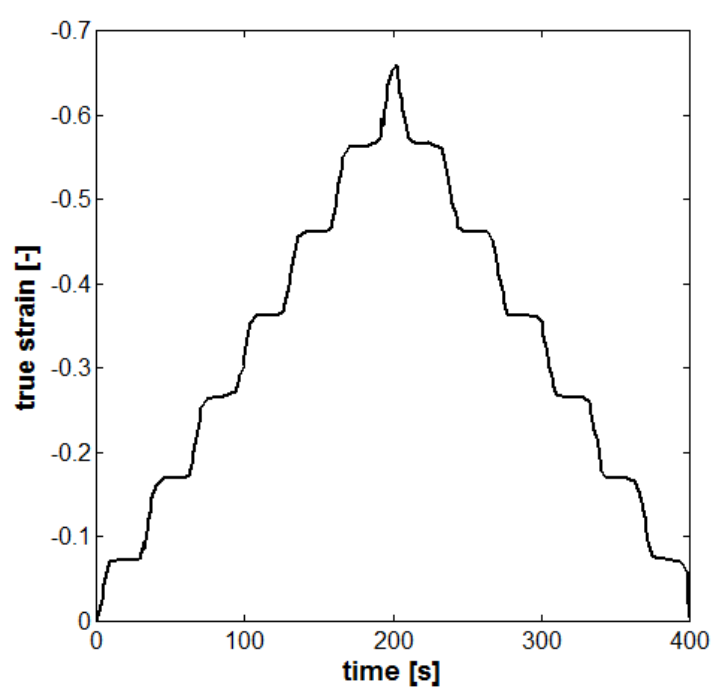

a)

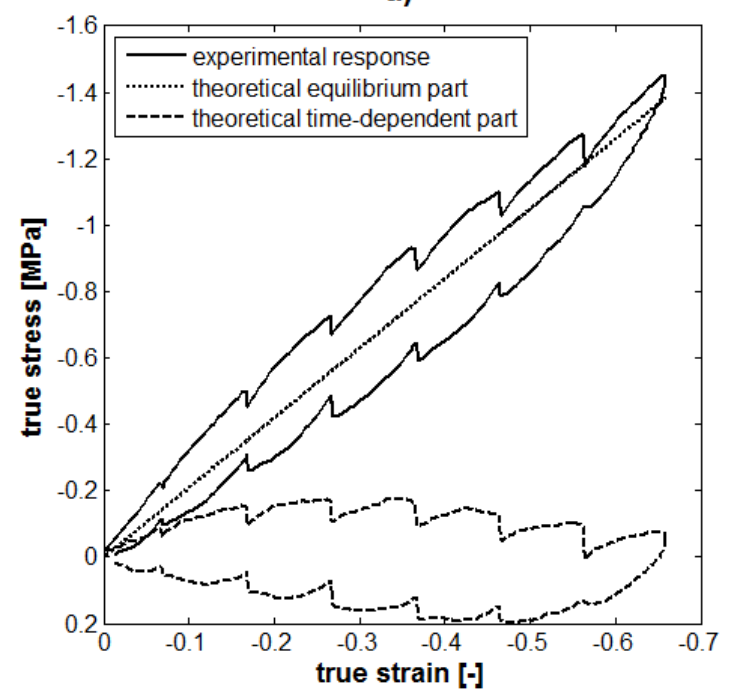

b)

Fig. 2. Presentation of the main concept behind Bergström-Boyce model: a) load history, b) decomposition of true stress vs true strain curves into equilibrium and time dependent part. Modified plots are based on the original work from (Bergström, 1999)

The Bergström-Boyce model (Bergström and Boyce, 1998), further referred as BB model, is a constitutive model that allows to predict nonlinear and time-dependent response of elastomers. It bases on previous advancements in areas of viscosity of polymers and hyperelasticity, but tailors them into a new conception. The model is based on the assumption, that mechanical response of an elastomer consists of two parts: elastic equilibrium and timedependent parts. This assumption comes from the experiment described in (Bergström, 1999) and presented in Fig. 2. An uniaxial compression test was conducted on a cylindrical specimen with additional relaxation periods, where strain was held at a constant value. It was observed that during relaxation, both on loading and unloading curve, recorded stress values were drifting to a theoretical line, which could be interpreted as a response in equilibrium conditions. This lead to assumption that elastomer, which is a network of polymer chains, could be treated as a system consisting of pure elastic network $A$ and viscoelastic network $B$. It must be that noted this is a kind of idealization for the purpose of modelling. Those networks are responsible for, respectively, equilibrium and time-dependent parts of elastomer behaviour (Fig. $2 \mathrm{~b}$ ). If this system should be depicted using mechanical equivalents, it would look like in Fig. 3 which is similar to classic rheological Zener model (also named standard solid model). Network $A$ is presented as single spring and network $B$ as damper and spring connected in series. This kind of approach can be found in other material models (Kießling, 2016). Such composition has important features as it allows to portray material creep and stress relaxation phenomenon, both of which occur in elastomers and are important for a complete description of mechanical response for this type of material.

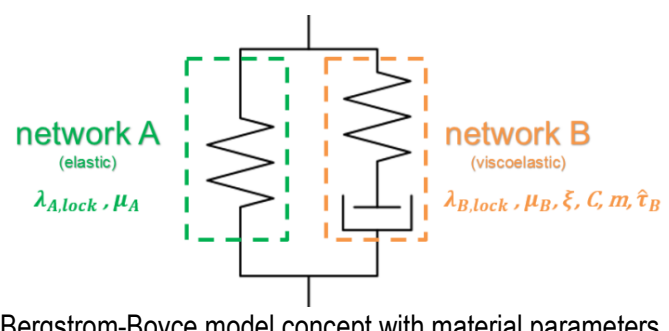

Fig. 3. Bergstrom-Boyce model concept with material parameters for each of the networks

In the present paper only strains due to mechanical deformation are considered, because all experiments have been conducted at constant, room temperature ca. $23^{\circ} \mathrm{C}$. Assumption of material incompressibility is also made as it simplifies the study without leading to significant mistakes.

Classic hyperelastic material models can be illustrated as a simple spring with nonlinear characteristic, dependent on specific model. Therefore, it seems to be appropriate and correct to use one of known hyperelastic model to describe spring elements shown in Fig. 3. Those models usually have a form of an equation for strain energy density as a function of invariants of deformation tensor, which in most cases is based on phenomenological observations. Thus it is expected that application range of such models would be limited to specific materials or load modes. In the BB model author chose to use eight-chain (EC) model (Arruda and Boyce, 1993) which is motivated by a behaviour of elastomers on microstructural level. It assumes that an elementary volume of elastomer element has the shape of cube. It contains eight polymer chains attached to each of vertices of the cube and converging at its centre point. Edges of cube are orientated according to direction of principal stretches. This leads to the following equation for effective distortional stretch:

$\bar{\lambda}=\sqrt{\frac{\lambda_{1}{ }^{2}+\lambda_{2}{ }^{2}+\lambda_{3}{ }^{2}}{3}}$ 
which is used next in the expression for stress:

$\boldsymbol{\sigma}=\frac{\mu}{\bar{\lambda}} \cdot \frac{\mathcal{L}^{-1}\left(\frac{\bar{\lambda}}{\lambda_{\text {lock }}}\right)}{\mathcal{L}^{-1}\left(\frac{1}{\lambda_{\text {lock }}}\right)} \cdot \boldsymbol{b}^{\prime}$

where: $\sigma$ - Cauchy stress tensor, $\mu$ - shear modulus [MPa], $\mathcal{L}(\mathrm{x})=\operatorname{coth}(\mathrm{x})-\frac{1}{\mathrm{x}}-$ Langevin function, $\mathcal{L}^{-1}(\mathrm{x})-$ inverse Langevin function, $\lambda_{\text {lock }}-$ maximum possible stretch of a polymer chain [-], b' - deviator of left Cauchy-Green strain tensor; its symbol is small letter $b$ to avoid confusion with network $B$.

The EC model is potentially more versatile than its phenomenological counterparts, because it proposes rationally justified mechanism of deformation. Material parameters $\mu, \lambda_{\text {lock }}$ have to be identified experimentally. It seems that EC model is correct choice for a foundation of a more complex BB model.

Basic concept used in BB model is based on works of de Gennes (Gennes, 1971) who described motion of polymer chains inside network and called it reptation (from latin reptate - to creep). Theory of reptational motion was later further developed in (Doi and Edwards, 1986). Bergstrom brought those previous achievements closer to practical applications in his BB model (Bergström, 1999) in the form of an equation for viscous rate:

$\dot{\gamma}_{B}=\gamma_{0} \cdot\left(\overline{\lambda_{B v}}-1+\xi\right)^{C} \cdot\left(\frac{\tau_{B}}{\sqrt{2} \cdot \hat{\tau}_{B}}\right)^{m}$

where: $\gamma_{0}=1\left[\frac{1}{s}\right]$ - constant for units agreement, $\overline{\lambda_{B v}}$ - viscous component of effective distortional stretch for network $B$, $\xi$ - material constant [-], $C$ - material constant [-], $\frac{\tau_{B}}{\sqrt{2}}=\frac{\left\|\sigma_{B}^{\prime}\right\|_{F}}{\sqrt{2}}-$ equivalent shear stress for network $B$ [MPa], $\boldsymbol{\sigma}_{B}^{\prime}$ - deviator of stress tensor for network $B,\|\ldots\|_{F}-$ Frobenius norm of a matrix, $\hat{\tau}_{B}$ - material constant $[\mathrm{MPa}], m$ - material constant [-].

To obtain specific stretch rate for viscous part the following expression (Bergström, 2015) should be used:

$\dot{\boldsymbol{F}}_{B v}=\dot{\gamma}_{B} \cdot\left(\boldsymbol{F}_{B e}\right)^{-1} \cdot \frac{\boldsymbol{\sigma}_{B}^{\prime}}{\left\|\boldsymbol{\sigma}_{B}^{\prime}\right\|_{F}} \cdot \boldsymbol{F}_{B e} \cdot \boldsymbol{F}_{B v}$

where: $\dot{\boldsymbol{F}}_{B v}$ - deformation rate tensor for viscous part of network $\mathrm{B}, \boldsymbol{F}_{B e}$ - deformation tensor for elastic part of network B, $\boldsymbol{F}_{B v}-$ deformation tensor for viscous part of network $B, \boldsymbol{\sigma}_{B}^{\prime}$ - deviator of the stress tensor of network $B$.

Characterization of viscoelastic behavior of polymer network, like in case of EC model, is a result of analysis of this phenomenon and its mechanisms which has concluded in the successful attempt to describe it with mathematical formulas. Therefore it can be stated that whole BB model, both in elastic and viscoelastic part, is a model which is strongly connected with polymer mechanics theory. Thus it is versatile and has big potential for employing it for FEM computations of mechanical systems (Dal and Kaliske, 2009). BB model seems to be useful tool for modelling elastomer elements as there are publications describing its successful applications (Chouinard et al., 2009; Ghoreishy, 2014).

The BB model in the version presented in this paper has eight parameters, which have to be estimated in order to utilize it. Results of experimental tests based on which mentioned parameters were evaluated will be presented in the next section of present paper.

\section{MATERIAL PARAMETERS IDENTIFICATION}

Typical procedure of estimation of material parameters in hyperelastic model requires conducting a series of experimental test on standard specimens. Those tests are usually uniaxial tension, compression or simple shear. Empirically obtained force-displacement (stress-strain) curves are subsequently used for the model calibration. Next, the equation of theoretical curve for specific test should be derived from general model and fitted to empirical one. Sometimes, depending on model, one type of test is not enough to properly estimate material characteristics. Therefore, the general principle says that the more load modes are tested, the better performance of the model can be expected. This is true especially in case of phenomenological models. However, in case of BB model, because of reasons accented in previous section, in most cases one type of test should be enough to obtain satisfying results with this model.

Number of parameters which can be estimated in case of BB model is eight and it makes this model a bit sophisticated and difficult to calibrate. Referring to its original author (Bergström, 1999) some of those parameters can be specified before actual experiments. It is suggested that $\lambda_{\text {lock }}$ for both $A$ and $B$ networks can be assumed to be the same. Another one, $\xi$, is constant which task is to prevent singularity when $\overline{\lambda_{\mathrm{Bv}}} \rightarrow 1$ (undeformed state) and is usually taken as 0.0001 . This reduces number of model parameters to six and simplifies fitting operation. Even though, it is still a six dimensional optimization problem, because fitting can be also treated as minimization of difference between experimental and theoretical results where material parameters are decision variables. Possibility of getting stuck during optimization process in some local minima exists. Therefore it is advisable to use algorithms incorporating stochastic elements, which are able to break out of local minimas. The estimation of BB model parameters in this paper was carried out with a help of a genetic algorithm.

Theoretical formula for uniaxial compression of cylindrical specimen according to $B B$ model consists of three basic equations: stress of the network $A$, stress of the network $B$ and viscous stretch rate of the network $B$. All following formulas were derived for uniaxial loading of incompressible material. Deformation gradient $F$ for this specific loading mode is:

$\boldsymbol{F}=\left[\begin{array}{ccc}\lambda & 0 & 0 \\ 0 & \frac{1}{\sqrt{\lambda}} & 0 \\ 0 & 0 & \frac{1}{\sqrt{\lambda}}\end{array}\right]$

where: $\mathbf{F}$ - deformation gradient, $\lambda$ - principal stretch along compression axis [-].

Deformation gradient $\mathrm{F}$ can be written also as the product of elastic and viscous parts:

$\boldsymbol{F}=\boldsymbol{F}_{A}=\boldsymbol{F}_{B}=\boldsymbol{F}_{B e} \cdot \boldsymbol{F}_{B v}$

Stress in both networks for uniaxial load, per EC model is expressed by (Bergström, 1999):

$\sigma_{A}=\frac{\mu_{A}}{\overline{\lambda_{A}}} \cdot \frac{\mathcal{L}^{-1}\left(\frac{\overline{\lambda_{A}}}{\lambda_{A, l o c k}}\right)}{\mathcal{L}^{-1}\left(\frac{1}{\lambda_{A, l o c k}}\right)} \cdot\left(\lambda_{A}^{2}-\frac{1}{\lambda_{A}}\right)$ 
$\sigma_{B}=\frac{\mu_{B}}{\overline{\lambda_{B e}}} \cdot \frac{\mathcal{L}^{-1}\left(\frac{\overline{\lambda_{B e}}}{\lambda_{B, l o c k}}\right)}{\mathcal{L}^{-1}\left(\frac{1}{\lambda_{B, \text { lock }}}\right)} \cdot\left(\lambda_{B e}^{2}-\frac{1}{\lambda_{B e}}\right)$

and stretch rate for viscous part in network $B$ :

$\dot{\lambda}_{B v}=\dot{\gamma}_{0} \cdot\left(\overline{\lambda_{B v}}-1+\xi\right)^{C} \cdot\left(\frac{1}{\sqrt{3}} \cdot \frac{\sigma_{B}}{\hat{\tau}_{B}}\right)^{m} \cdot \sqrt{\frac{2}{3}} \cdot \lambda_{B v}$

where: $\lambda_{A}=\lambda_{B}=\lambda, \quad \overline{\lambda_{A}}=\sqrt{\frac{\lambda_{A}{ }^{2}+\frac{2}{\lambda_{A}}}{3}}, \quad \overline{\lambda_{B v}}=\sqrt{\frac{\lambda_{B v}{ }^{2}+\frac{2}{\lambda_{B v}}}{3}}$, $\overline{\lambda_{B e}}=\sqrt{\frac{\lambda_{B e^{2}+\frac{2}{\lambda_{B e}}}^{3}}{3}}, \lambda_{B e}$ - elastic part of stretch in the network $\mathrm{B}$,

$\lambda_{B v}$ - viscous part of stretch in the network $B, \lambda_{A, l o c k}=$ $\lambda_{B, \text { lock }}=\lambda_{\text {lock }}$.

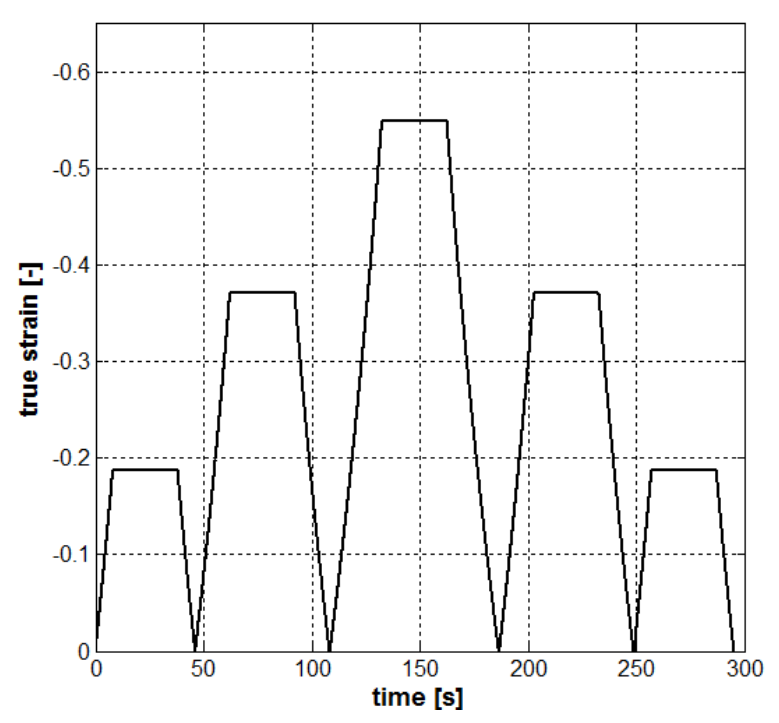

Fig. 4. Load sequence used for the calibration of BB model

Experimental tests were conducted on cylindrical specimens of $30 \mathrm{~mm}$ diameter and $14 \mathrm{~mm}$ height. Specimens were made from SBR (styrene-butadiene rubber) in two hardness variants: 50 ${ }^{\circ} \mathrm{ShA}$ and $70^{\circ} \mathrm{ShA}$ (Shore hardness type A). It should be noted that also a $90^{\circ} \mathrm{ShA}$ SBR rubber was initially planned for experiments but it proved to be too stiff for its effective utilisation in metal-elastomer spring thus it was omitted. Samples underwent uniaxial compression on universal testing machine. Registered results were later used for evaluating parameters of $\mathrm{BB}$ model.

In Fig. 4 is shown a time history of applied load in test which was utilized to calibrate BB model. It consists of five consecutive cycles with changing maximum true strain. At peak strain in each of them there is a relaxation period where strain is held at a constant value. Characteristic feature of elastomers is stress relaxation in time. Incorporating relaxation effect into material testing of elastomers make it easier to describe viscoelastic effects using BB model. Before executing calibration test from Fig. 4 rubber samples underwent mechanical conditioning which allowed to stabilize their mechanical response and significantly reduce Mullins effect. Fitting results are shown in Fig. 5. Received results presents satisfactory coincidence with experimental tests. Calibration test performed for an only single strain rate was sufficient because next experiments on metal-elastomer springs were conducted with relatively low speeds. Elastomer hardening due to deformation rate magnitude which was employed during springs tests (far below $1 \mathrm{~Hz}$ ) was not significant.
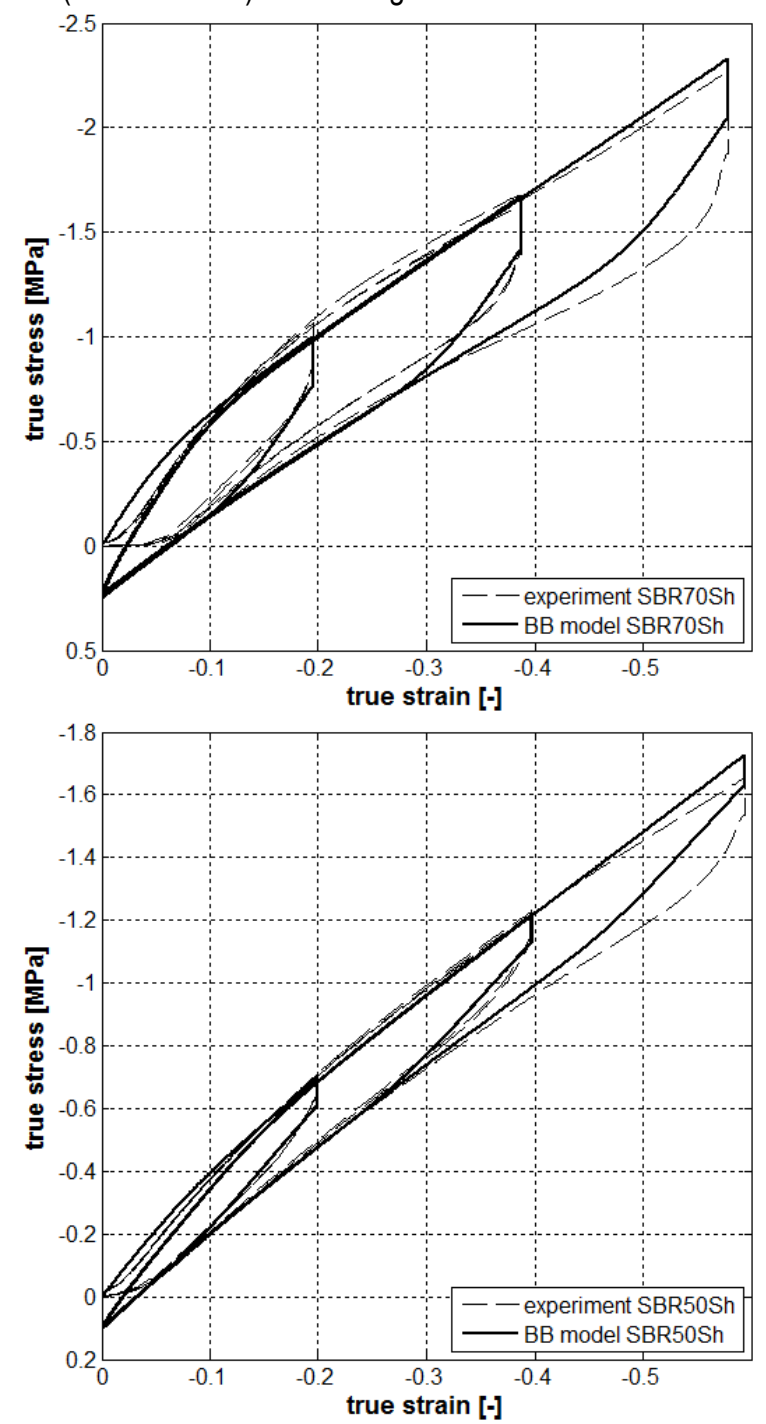

Fig. 5. Results of fitting BB model to experimental curves for materials SBR $70^{\circ}$ Sh and SBR $50^{\circ}$ Sh

\section{EXPERIMENTAL TESTS AND FEM SIMULATIONS}

To validate results of the FEM simulations there must be conducted a series of experimental tests on metal-elastomer springs which would serve later as a reference for assessment. A single elastic joint (Fig. 1a) is a torsion spring, thus it must be loaded with moment of force or angular displacement. Therefore, a simple apparatus which allows to adapt a typical universal test machine to perform such kind of experiment was designed and assembled. It is shown in Fig. 6 and it consists of rack (2) and pinion gear (3) that is fixed on the shaft (4). It allows to convert linear to angular motion (comparatively force to moment). Shaft itself was supported by two bearings (6) that were fixed to the device frame. Tested joint (5) was pushed onto shaft and then fixed in place. Load has been applied through testing machine jaws (1) which hold rack and allows to move it vertically. Force inflicted on rack and its displacement were recorded by a testing machine software and were converted later to angle and moment respectively. The 
rotary inertia of the whole system does not influence adversely the results of experiments due to quasi-static character of displacements.

Three types of tests with varying load sequence were performed. Plots showing angle vs time history for each of them are shown in Fig. 7. Fig. 7a represents a symmetrical cyclic loading with $30^{\circ}$ rotation clockwise and counter-clockwise, consisting of three full cycles. It explores the whole rotational working range of the joint. Second load sequence, presented in Fig. 7b, is in close proximity to the loads which undergo metal-elastomer springs in the most of applications. It starts with initial load, $15^{\circ}$ in this case, and then oscillates around it with amplitude equal to $5^{\circ}$. Initial static load represents the force associated with the weight of the machine. Later comes the working load in the form of vibration/oscillations. This test involves full four load cycles. The last type of loading sequence, presented on Fig. 7c, consists of three consecutive cycles with increasing true strain value.
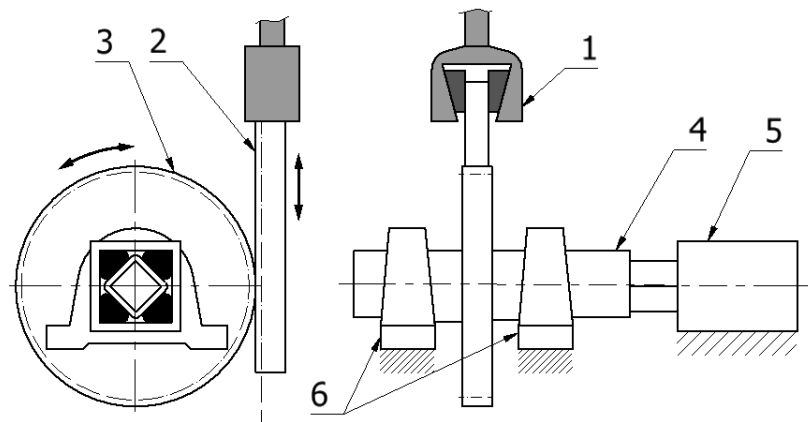

Fig. 6.Diagram of apparatus for testing metal-elastomer springs:

1 - grips, 2 - rack, 3 - pinion gear, 4 - shaft, 5 - tested spring, 6 - support bearings

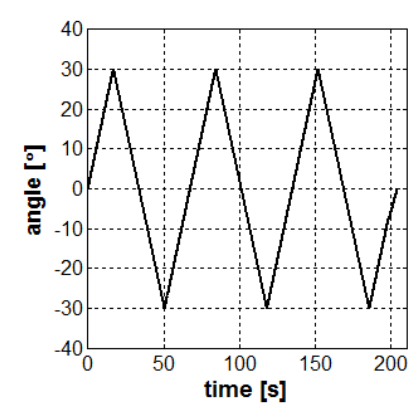

a)

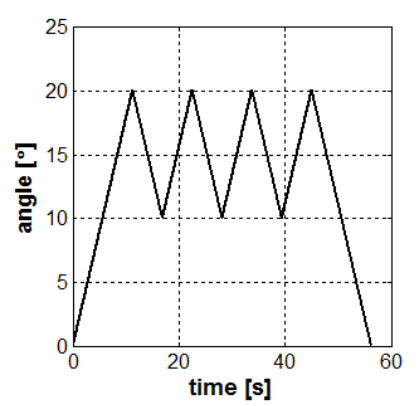

b)

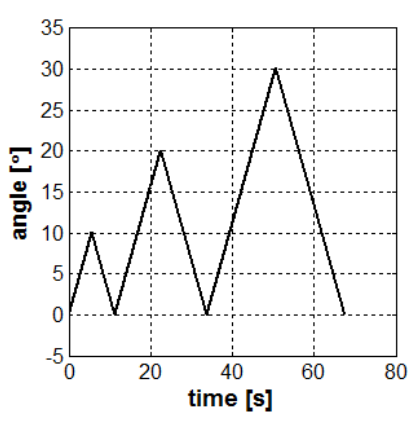

c)

Fig. 7. Load sequences used in FEM simulations and experimental test of metal-elastomer springs

FEM simulations were conducted with the help of Ansys Mechanical APDL. The prepared solid model consists, like its real-life counterpart, of three parts: outer square tube, elastomer cylinder and inner square tube. Dimensions of spring's elements are shown in Fig. 8. Elastomer elements before assembly had cylinder shape of $12 \mathrm{~mm}$ in diameter and $40 \mathrm{~mm}$ length. Due to three symmetry planes which can be found in the considered spring, only $1 / 8$ of assembly model was considered in simulations, replacing missing parts of model with proper boundary conditions (Fig. 8). Boundary conditions for the simulation were assumed to be identical like in experimental tests, i.e. element (1) in Fig. 9 was fixed and element (3) was constrained in such a way, that it could only rotate around main axis, as it is shown in Fig. 9. The result of each simulation was a moment reaction on element (3) as function of time and rotation angle. Bodies (1) and (3) were treated as rigid and (2) as flexible.
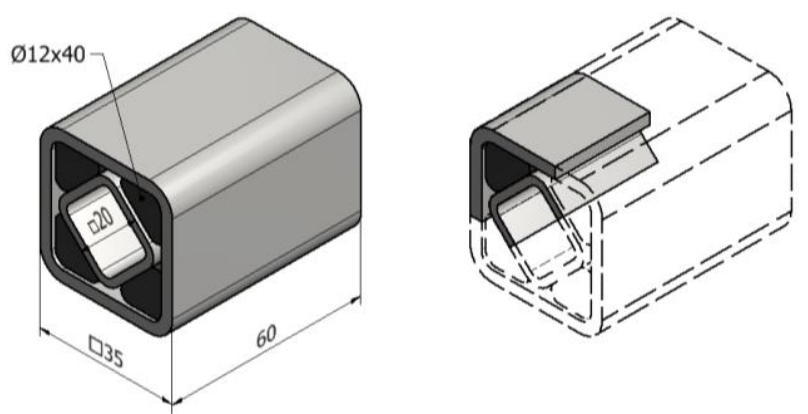

Fig. 8. Reduction of model size due to existing symmetry planes
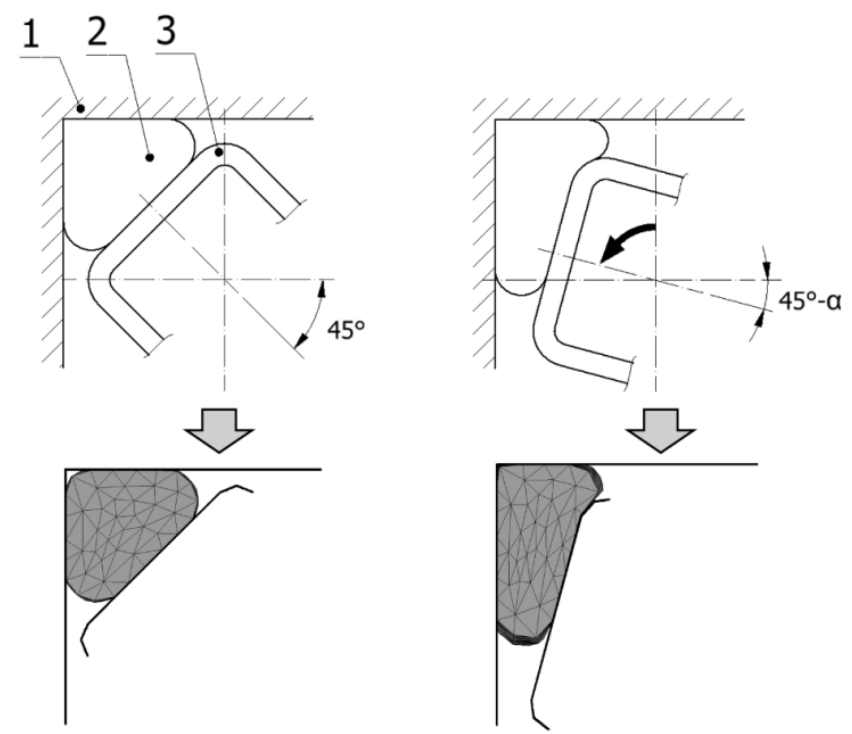

Fig. 9. Schematic of FEM simulation. 1 - outer square tube, 2 - elastomer, 3 - inner square tube

Mechanical response of metal-elastomer springs, due to rubber properties, is slightly changing during initial load cycles, mainly because of softening effect. Thus, before actual experiments, springs were mechanically conditioned to stabilize their response. Thanks to that the third cycle from experimental load sequences in Fig.7a and Fig. 7b was representing equilibrium state response and therefore was taken in to the comparison between experimental results and FEM simulations. 


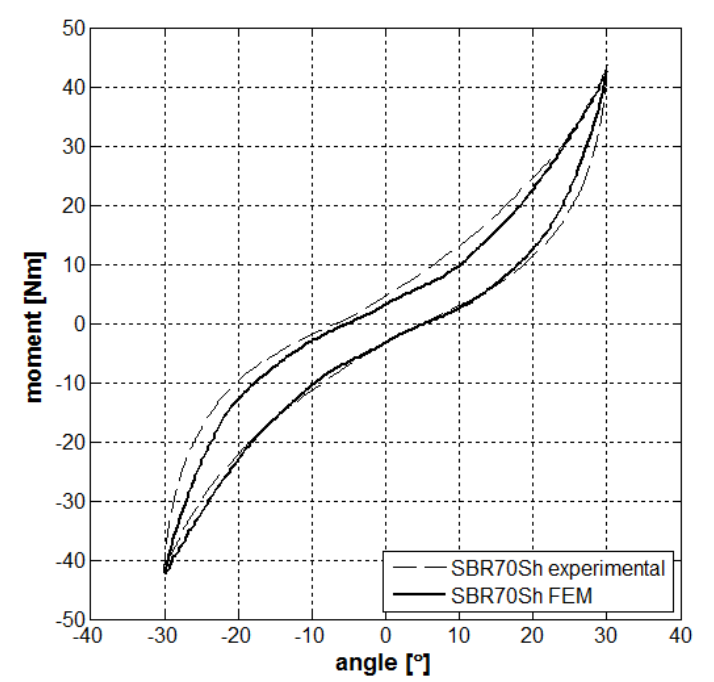

load sequence a for SBR $70^{\circ} \mathrm{Sh}$

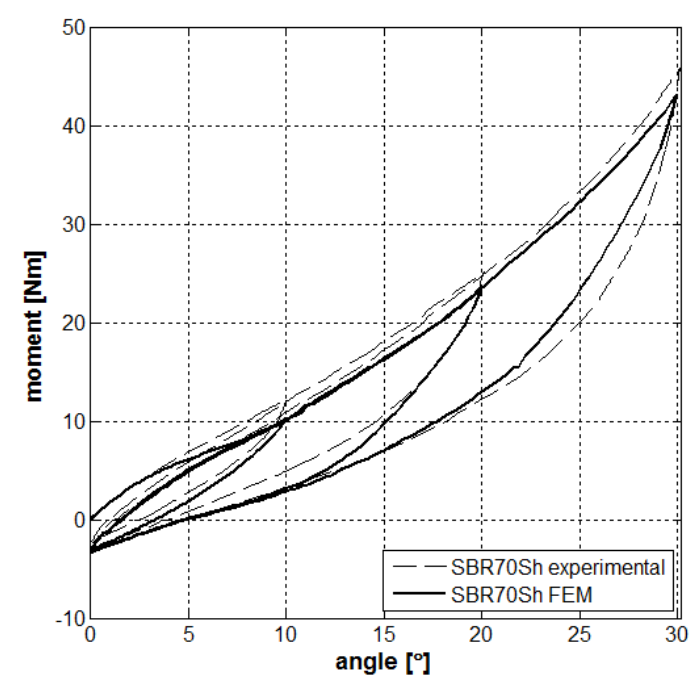

load sequence $\mathbf{c}$ for $\mathrm{SBR} 70^{\circ} \mathrm{Sh}$

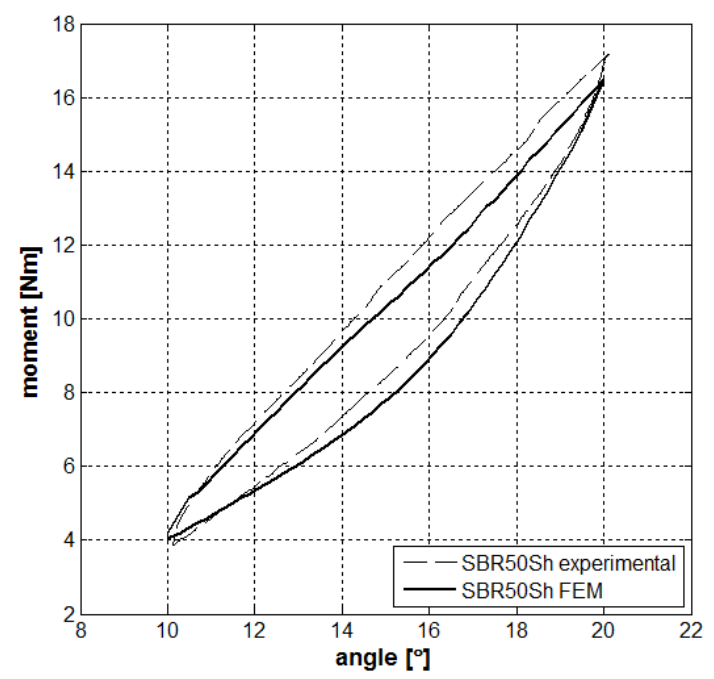

load sequence $\mathbf{b}$ for SBR $50^{\circ} \mathrm{Sh}$

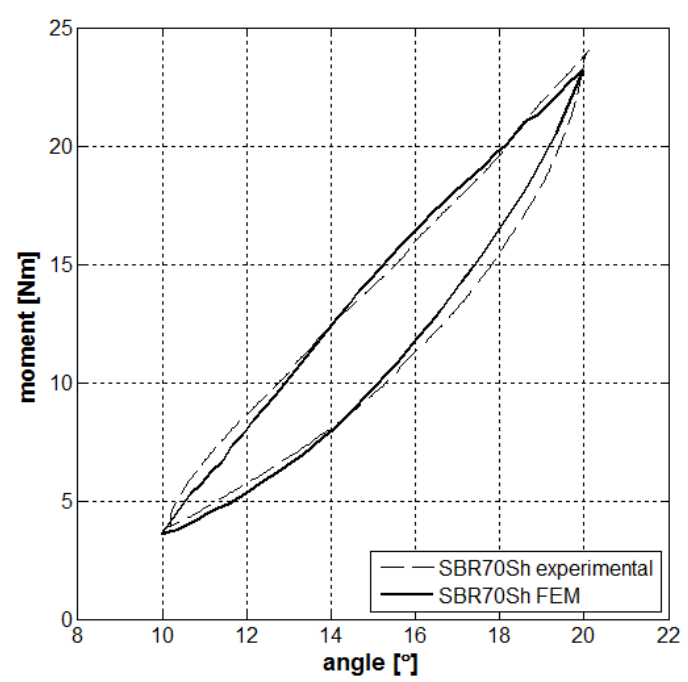

load sequence $b$ for $\operatorname{SBR} 70^{\circ} \mathrm{Sh}$

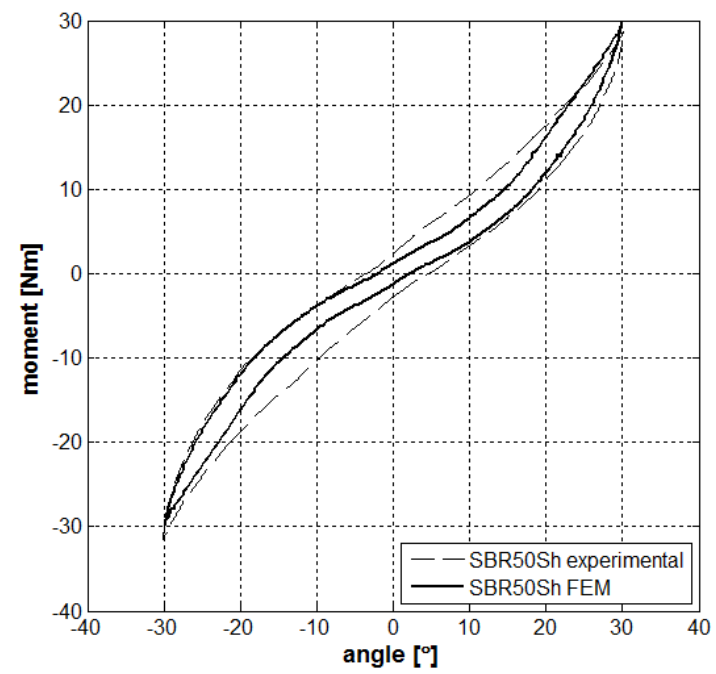

load sequence a for SBR $50^{\circ} \mathrm{Sh}$

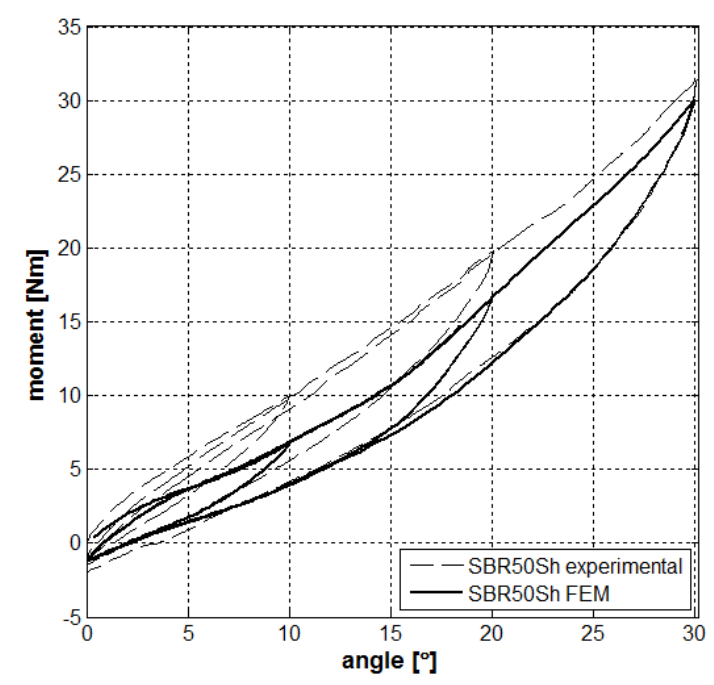

load sequence $\mathrm{c}$ for $\mathrm{SBR} 50^{\circ} \mathrm{Sh}$

Fig. 10. Comparison between experimental tests and FEM simulations results 


\section{RESULTS}

Comparison between experimental and FEM simulation results is shown in Fig. 10. Considering first specimen, i.e. spring containing elastic elements made of SBR $70^{\circ} \mathrm{ShA}$ rubber, it can be seen, that numerical simulation provides quite accurate results, coincident with experimental characteristics. Maximum values of moment for each of load sequences from Fig. 7a-c are very similar, with slight deviation around $2 \%$ to $6 \%$. The shape of the experimental curves are well represented by the simulated ones. However, slight deviations in the size of elastic hysteresis can be observed in each sequence. Sample with softer rubber, SBR $50^{\circ} \mathrm{Sh}$, shows at the other hand more significant discrepancy. General shape of FEM curves for load sequence from Fig. 7a and 7c have a slightly lower compliance with experimental test results than the ones for the tougher rubber It can also be observed, that hysteresis loop for load sequence in Fig.7b, obtained from experimental tests is significantly narrower than the area obtained from FEM simulations.

Considering that FEM simulation and experiment conditions were identical for both rubber types, the source of noted differences is the material itself. From mechanical point of view these two rubber compositions differ only by Shore hardness. However, rubber hardness is a collective effect of its chemical composition i.e. base rubber blend and additives and fillers. It can be observed that BB model exhibits lower accuracy, when describing behaviour of soft rubber blends in complicated loading cases.

\section{CONCLUSIONS}

This paper presents a numerical FEM modelling of metalelastomer spring nonlinear response for low-rate deformations. It describes an outline of Bergstrom-Boyce hyperelastic model used later in numerical calculations and a method for its material parameters identification. Experimental tests were performed for two types of SBR rubber with different hardness, i.e. $70^{\circ} \mathrm{ShA}$ and $50^{\circ} \mathrm{ShA}$. Load-deformation curves obtained from FEM simulations were compared with empirical results acquired with the help of custom made apparatus on universal testing machine.

The main aim of this work was to evaluate possibilities of modelling of metal-elastomer springs at low deformation rates. For the reason that rubber elements undergo large deformations during assembly and later during operation of such spring, analytical means of description seemed to be difficult to apply in this case. Alternative solution was FEM simulation. Obtained numerical results were assessed by comparing them with curves obtained from empirical tests. Simulations performance were different for both of tested materials. Metal-elastomer spring containing elastic elements made of SBR $70^{\circ} \mathrm{ShA}$ behaved similarly during experiment and FEM simulation. There was slight difference in peak values of moment and elastic hysteresis but in general it can be summarized as successful attempt. Evaluation of simulation for SBR $50^{\circ} \mathrm{ShA}$ is notably worse than in previous case. The main problem which occurred is visible discrepancy in elastic hysteresis reproduction.

Hardness of the rubber used in considered metal-elastomer springs which are available on the market is around $70^{\circ} \mathrm{ShA}$. Modifications of the spring stiffness is in most cases realised by changing dimensions of rubber elements, while rubber hardness remain unchanged. Softer SBR blend was included in this work for the purpose of evaluation performance of BB model in FEM simulations.
Further work should concern influence of deformation rate on mechanical characteristics of metal-elastomer springs and possibilities of modelling it with FEM.

\section{REFERENCES}

1. Arruda E.M., Boyce M.C. (1993), A three-dimensional constitutive model for the large stretch behavior of rubber elastic materials, Journal of the Mechanics and Physics of Solids, 41 (2), 389-412.

2. Bergström J.S. (1999), Large strain time-dependent behavior of elastomeric materials, Ph.D. thesis, MIT.

3. Bergström J.S. (2015), Mechanics of solid polymers: theory and computational modeling, William Andrew, San Diego, USA.

4. Bergström J.S., Boyce M.C. (1998), Constitutive modeling of the large strain time-dependent behavior of elastomers, Journal of the Mechanics and Physics of Solids, 46, 931-954.

5. Chouinard, P., Proulx, S., Lucking Bigué J.P., Plante, J. (2009), Design of an antagonistic bistable dielectric elastomer actuator using the Bergstrom-Boyce constitutive viscoelastic model, presented at 33rd Mechanisms and Robotics Conference, 2009, San Diego, CA, USA.

6. Cieplok G. (2009), Verification of the nomogram for amplitude determination of resonance vibrations in the run-down phase of a vibratory machine, Journal of Theoretical and Applied Mechanics, 47, 295-306.

7. Dal H., Kaliske M. (2009), Bergström-Boyce model for nonlinear finite rubber viscoelasticity: theoretical aspects and algorithmic treatment for the FE method, Computational Mechanics, 44, 809-823.

8. Diego S., Casado J. A, Carrascal I., Ferreno D., Cardona, J., Arcos R. (2017), Numerical and experimental characterization of the mechanical behavior of a new recycled elastomer for vibration isolation in railway applications, Construction and Building Materials, 134, 18-31.

9. Doi M., Edwards S.F. (1986), The theory of polymer dynamics. Oxford University Press, Oxford.

10. Gennes P.G. (1971), Reptation of a polymer chain in the presence of fixed obstacles, The Journal of Chemical Physics, 55 (2), 572-579.

11. Gent A. N. (1996), A new constitutive relation for rubber, Rubber Chemistry and Technology, 69, 59-61.

12. Ghoreishy M.H.R., Firouzbakht M., Naderi G. (2014), Parameter determination and experimental verification of Bergström-Boyce hysteresis model for rubber compounds reinforced by carbon black blends. Materials and Design, 53, 457-465.

13. Ghoreishy M.H.R., Naderi G., Roohandeh B. (2015), An experimental investigation on the degradation effect of ozone on hyperelastic behavior of an NR/BR blend, Iranian Polymer Journal, 24(12), 1015-1024.

14. Hossain M., Vu D.K., Steinmann P. (2012), Experimental study and numerical modelling of VHB 4910 polymer, Computational Materials Science, 59, 65-74.

15. Kießling R., Landgraf R., Scherzer R., Ihlemann J. (2016), Introducing the concept of directly connected rheological elements by reviewing rheological models at large strains, International Journal of Solids and Structures, 97-98, 650-667.

16. Mooney M. (1940), A theory of large elastic deformation, Journal of Applied Physics, 11(9), 582-592.

17. Neidhart H. (1951), Elastic joints, US patent 2712742.

18. Sikora W., Michalczyk K., Machniewicz T. (2016), A study of the preload force in metal-elastomer torsion springs, Acta Mechanica et Automatica, 10(4), 300-305.

19. Yeoh O.H. (1993), Some forms of the strain energy function for rubber, Rubber Chemistry and Technology, 66(5), 754-771.

Acknowledgements: This work is supported by AGH University of Science and Technology under research program no. 15.11.130.598. 